\title{
A numerical study of thin film flow of a non-Newtonian fluid on a vertically moving belt using variational iteration approach
}

\author{
A. A. Farooq $^{1 *}$, Belal Batiha ${ }^{2}$, A. M. Siddiqui ${ }^{3}$ \\ ${ }^{1}$ COMSATS University of Science and Technology, Abbottabab, Pakistan \\ ${ }^{2}$ Higher Colleges of Technology, Abu Dhabi Men's College, UAE \\ ${ }^{3}$ Department of Mathematics, York Campus, Pennsylvania State University, USA \\ *Corresponding author E-mail: aliahmedfarooq@yahoo.com
}

\begin{abstract}
This paper provides an investigation regarding the modeling and analysis of a thin film flow of an Oldroyd 8-constant fluid on a vertically moving belt. The governing nonlinear problem is solved by using Variational Iteration Method (VIM). The results of the present method are then compared with those obtained by Adomian Decomposition Method (ADM) and an excellent agreement is observed. This comparison reveals that VIM may be considered as an efficient alternative method for solving nonlinear problems arising in non-Newtonian fluid mechanics. Expressions for some important physical quantities such as volume flux, average velocity, the belt speed for the lifting of the non-Newtonian fluid are also derived.
\end{abstract}

Keywords: Adomian decomposition method, moving belt, nonlinear equation, thin film flow, variational iteration method.

\section{Introduction}

In the recent years, the studies of thin film flows of non-Newtonian fluids have received considerable attention by many researchers, see for instance [1-4], and the references therein. This is perhaps due to their several applications in nonlinear sciences and engineering industries. The differential equations that arise when modeling non-Newtonian fluids in thin film flow problems are in general highly nonlinear and complicated. The analytical study of such type of nonlinear problems is important not only because of their technological significance but also due to the interesting mathematical features presented by the governing differential equations of the flow. It is well known that most of these types of problems do not have an exact solution. Therefore, these equations should be treated by using some numerical or analytical methods. Apart from numerical methods, several analytical techniques such as HAM, HPM, ADM and VIM are proposed to find approximate analytical, and if possible in closed form, solutions of such nonlinear equations, see [5-9], and the references therein.

The basic motivation of this paper is to apply the VIM and the ADM to find the approximate analytical solution of a highly nonlinear differential equation that arises in the thin film flow problems of a non-Newtonian Oldroyd 8-constant fluid lifting on a moving belt. The VIM as a powerful analytical technique was first introduced by He and has been used by many mathematicians to solve various nonlinear equations [10-14]. This method gives rapidly convergent successive approximations of the exact solutions if such solution exists. For this problem, the governing equation is highly nonlinear whose exact solution is very difficult, a few number of approximations can be used for numerical purposes with high degree of accuracy. For comparison the same problem is also solved by ADM. The results show that difference between the two solutions is negligible. This comparison is benched-marked against a numerical solution. A considerable amount of research work has been conducted recently in applying these methods to a class of linear and nonlinear problems see [10-14]. However, Variational iteration Method has an advantage over Adomian Decomposition Method that it solves nonlinear problems without using Adomian polynomials. For the convergence criteria and error estimation of VIM, see [13, 14]. 


\section{Governing Equations}

The fundamental equations governing the flow of an incompressible fluid, neglecting the thermal effects are

$\nabla . \mathrm{V}=0$,

$\rho \frac{d \mathrm{~V}}{d t}=-\nabla p+\rho \mathrm{f}+\nabla . \mathrm{S}$

where $\mathrm{V}=(u, v, w)$ is the velocity vector, $\rho$ is the constant density, $\mathrm{f}=\left(\mathrm{f}_{1}, \mathrm{f}_{2}, \mathrm{f}_{3}\right)$ is the body force per unit mass, $\frac{d}{d t}$ is the material time derivative, $p$ is the dynamic pressure and $\mathrm{S}$ is the extra stress tensor, which for Oldroyd 8-constant fluid is defined as [15]

$\mathrm{S}+\lambda_{1} \stackrel{\nabla}{\mathrm{S}}+\frac{\lambda_{3}}{2}\left(\mathrm{~A}_{1} \mathrm{~S}+\mathrm{SA}_{1}\right)+\frac{\lambda_{5}}{2}(t r \mathrm{~S}) \mathrm{A}_{1}+\frac{\lambda_{6}}{2}\left[\left(t r \mathrm{SA}_{1}\right)\right] \mathrm{I}$
$=\mu\left[\mathrm{A}_{1}+\lambda_{2} \stackrel{\nabla}{\mathrm{A}}_{1}+\lambda_{4} \mathrm{~A}_{1}^{2}+\frac{\lambda_{7}}{2}\left[\left(t r \mathrm{~A}_{1}^{2}\right)\right] \mathrm{I}\right]$

where $\mathrm{I}$ is the identity tensor and $\mu$ and $\lambda_{i}(i=1,2, \ldots, 7)$ are the material constants of the fluid. The contravariant convected derivative is defined as

$\stackrel{\nabla}{\mathrm{S}}=\frac{d \mathrm{~S}}{d t}-\left[(\nabla \mathrm{V})^{T} \mathrm{~S}+\mathrm{S}(\nabla \mathrm{V})\right]$

$\mathrm{A}_{1}=\frac{d \mathrm{~A}_{1}}{d t}-\left[(\nabla \mathrm{V})^{T} \mathrm{~A}_{1}+\mathrm{A}_{1}(\nabla \mathrm{V})\right]$

and the Rivilin-Ericksen tensor $\mathrm{A}_{1}$ is defined as

$\mathrm{A}_{1}=(\nabla \mathrm{V})+(\nabla \mathrm{V})^{T}$

where ${ }^{T}$ stands for transpose of the tensor. It should be noted that the model (3) includes the Oldroyd 6-constant fluid when $\lambda_{6}=\lambda_{7}=0 ;$ Oldroyd 3-constant fluid when $\lambda_{3}=\lambda_{5}=\lambda_{6}=\lambda_{7}=0 ;$ Maxwell fluid when $\lambda_{2}=\lambda_{3}=\lambda_{4}=\lambda_{5}=\lambda_{6}=\lambda_{7}=0$; the second grade fluid when $\lambda_{1}=\lambda_{3}=\lambda_{4}=\lambda_{5}=\lambda_{6}=\lambda_{7}=0, \mu \lambda_{2}=\alpha_{1}$ and the Newtonian fluid for $\lambda_{1}=\lambda_{2}=\lambda_{3}=\lambda_{4}=\lambda_{5}=\lambda_{6}=\lambda_{7}=0$.

\section{Formulation of the problem}

We consider a container filled with an Oldroyd 8-constant fluid. A wide belt passes through this container, which moves vertically upward with a constant speed $V_{0}$. The moving belt picks up a thin film fluid of uniform thickness $\delta$. The gravity tries to make the fluid film drain down the belt. We assume that the flow is steady, laminar and uniform and the pressure is atmospheric pressure. We choose $x$-axis normal to the belt and $y$-axis is taken along the belt which is in upward direction.

The appropriate boundary conditions for the problem are

$v=V_{0} \quad$ at $x=0$ (no slip condition),

$S_{x y}=0 \quad$ at $x=\delta$ ( free surface),

where $S_{x y}$ is the shear stress component of the Oldroyd 8-constant fluid.

We seek velocity field and the extra stress tensor of the form

$\mathrm{V}=[0, v(x), 0], \quad \mathrm{S}=\mathrm{S}(x)$ 
When (9) is used in (1) and (2), the continuity Eq.(1) is identically satisfied and the momentum Eq.(2) is reduced to

$0=\frac{d S_{x x}}{d x}+\rho \mathrm{f}_{1}$

$0=\frac{d S_{x y}}{d x}+\rho \mathrm{f}_{2}$

where $\mathrm{f}_{1}$ and $\mathrm{f}_{2}$ are the components of body force in $x$ and $y$ directions, respectively. Since $y$-axis is taken to be in upward direction and the force due to gravity is in downward direction, so the above equations take the form

$0=\frac{d S_{x x}}{d x}$

$0=\frac{d S_{x y}}{d x}-\rho g$

Making use of Eqs.(4-6) and (9) in (3), we obtain the non-zero components of S as

$$
\begin{aligned}
S_{x x}= & \frac{\left(\mu \alpha_{1}\left(\lambda_{3}+\lambda_{6}\right)+\left(\mu\left(\lambda_{4}+\lambda_{2}\right)\left(1+\alpha_{2}\right)\right)\left(\frac{d v}{d x}\right)^{4}-\mu\left(\lambda_{3}+\lambda_{6}\right)\left(\frac{d v}{d x}\right)^{2}\right.}{1+\alpha_{2}\left(\frac{d v}{d x}\right)^{2}}, \\
S_{x y}= & \frac{\mu \frac{d v}{d x}+\mu \alpha_{1}\left(\frac{d v}{d x}\right)^{3}}{1+\alpha_{2}\left(\frac{d v}{d x}\right)^{2}}=S_{y x}, \\
S_{y y}= & \left(\mu\left(\lambda_{4}+\lambda_{7}-2 \lambda_{2}\right)-\mu\left(\lambda_{3}+\lambda_{6}-2 \lambda_{1}\right)\right)\left(\frac{d v}{d x}\right)^{2}-\left(\mu \alpha_{2}\left(\lambda_{4}+\lambda_{7}-2 \lambda_{2}\right)+\mu \alpha_{1}\left(\lambda_{3}+\lambda_{6}-2 \lambda_{1}\right)\right)\left(\frac{d v}{d x}\right)^{4}
\end{aligned}
$$

The constants $\alpha_{1}$ and $\alpha_{2}$ are defined by

$$
\begin{aligned}
& \alpha_{1}=\lambda_{1}\left(\lambda_{4}+\lambda_{7}\right)-\left(\lambda_{3}+\lambda_{5}\right)\left(\lambda_{4}+\lambda_{7}-\lambda_{2}\right)-\frac{\lambda_{5} \lambda_{7}}{2}, \\
& \alpha_{2}=\lambda_{1}\left(\lambda_{3}+\lambda_{6}\right)-\left(\lambda_{2}+\lambda_{5}\right)\left(\lambda_{3}+\lambda_{6}-\lambda_{1}\right)-\frac{\lambda_{5} \lambda_{6}}{2} .
\end{aligned}
$$

Using (15) in (13), the final form of the governing equation is given by

$$
\frac{d}{d x}\left[\frac{\mu \frac{d v}{d x}+\mu \alpha_{1}\left(\frac{d v}{d x}\right)^{3}}{1+\alpha_{2}\left(\frac{d v}{d x}\right)^{2}}\right]=\rho g
$$

The boundary conditions (7) and (8) are accordingly reduced to $v=V_{0} \quad$ at $x=0$ ( no slip condition),

$\frac{d v}{d x}=0 \quad$ at $x=\delta($ free surface $)$

Integrating once Eq.(17) with respect to $x$ and using the boundary condition (19), we obtain 
$\mu \frac{d v}{d x}+\mu \alpha_{1}\left(\frac{d v}{d x}\right)^{3}-\alpha_{2} \rho g(x-\delta)\left(\frac{d v}{d x}\right)^{2}=\rho g(x-\delta)$

$v=V_{0} \quad$ at $x=0$

Introduce the following dimensionless parameters

$v^{*}=\frac{v}{V_{0}}, x^{*}=\frac{x}{\delta}, \alpha_{1}^{*}=\frac{\alpha_{1}}{\left(\delta / V_{0}\right)}, \alpha_{2}^{*}=\frac{\alpha_{2}}{\left(\delta / V_{0}\right)}, m^{*}=\frac{\rho g \delta^{2}}{\mu N_{0}}$

Therefore, the governing differential equation for the problem with the boundary condition after dropping ${ }^{*}$ becomes

$\frac{d v}{d x}+\alpha_{1}\left(\frac{d v}{d x}\right)^{3}-m \alpha_{2}(x-1)\left(\frac{d v}{d x}\right)^{2}=m(x-1)$

$v=1 \quad$ at $x=0$.

It is noted that Eq.(22) along with one boundary condition (23) is a highly nonlinear first order ordinary differential equation. It is a well-posed problem but difficult to find its exact closed form solution. Therefore, we are interested in finding the approximate analytical solutions by using VIM and ADM.

\section{Solution by VIM}

To apply variational iteration method (VIM), we write equation $(\quad 22)$ in the form

$L(v)+N_{1}(v)+N_{2}(v)=f(x)$,

where $L(v)=\frac{d v}{d x}$ is the linear term, $N_{1}(v)=\alpha_{1}\left(\frac{d v}{d x}\right)^{3}$ and $N_{2}(v)=-m \alpha_{2}(x-1)\left(\frac{d v}{d x}\right)^{2}$ are nonlinear terms and $f(x)=m(x-1)$ is the forcing term. According to variational iteration method [8, 10, 14], we can construct a correction functional as follows

$v_{n+1}=v_{n}+\int_{0}^{x} \lambda(s)\left(L v_{n}(s)+N_{1} \tilde{v}_{n}(s)+N_{2} \tilde{v}_{n}(s)-f(s)\right) d s$,

where $\lambda(s)$ is a Lagrange multiplier, which can be identified optimally via variational theory. The subscript $n$ denotes the $n t h$ approximation and $\tilde{v}_{n}$ is considered as a restricted variation, that is, $\delta \tilde{v}_{n}=0$. The successive approximations $v_{n+1}, n \geq 0$ of the solution $v(x)$ are obtained upon using any selective function $v_{0}$, consequently, the solution is given by $v(x)=\lim _{n \rightarrow \infty} v_{n}$.

Employing the restricted variation in (25) we compute the Lagrange multiplier as

$\left.\delta v_{n+1}=\delta v_{n}+\delta \int_{0}^{x} \lambda(s)\left(L v_{n}(s)+N_{1}{\tilde{v_{n}}}_{(s)}+N_{2}{\tilde{v_{n}}}(s)\right)-f(s)\right) d s$

Integration by parts results the stationary conditions

$1+\left.\lambda\right|_{s=x}=0,\left.\lambda^{\prime}\right|_{s=x}=0$,

which in turn gives $\lambda=-1$.

Substituting this value of the Lagrange multiplier into the functional (26), we obtain the following iteration formula

$v_{n+1}(x)=v_{n}(x)-\int_{0}^{x}\left(\frac{d v_{n}}{d s}+\alpha_{1}\left(\frac{d v_{n}}{d s}\right)^{3}-m \alpha_{2}(s-1)\left(\frac{d v_{n}}{d s}\right)^{2}-m(s-1) s\right) d s, n \geq 0$ 
We start with initial approximation $v_{0}(x)=1$. The next iterates $v_{1}, v_{2}, v_{3}, \ldots$ are given below respectively

$$
\begin{aligned}
v_{1}(x)= & 1+\frac{m}{2}\left((x-1)^{2}-1\right), \\
v_{2}(x)= & 1+\frac{m}{2}\left((x-1)^{2}-1\right)+\frac{\alpha m^{3}}{4}\left((x-1)^{4}-1\right), \\
v_{3}(x)= & 1+\frac{m}{2}\left((x-1)^{2}-1\right)+\frac{\alpha m^{3}}{4}\left((x-1)^{4}-1\right)+\frac{\alpha \alpha_{3} m^{5}}{6}\left((x-1)^{6}-1\right) \\
& +\frac{\alpha^{2} \alpha_{4} m^{7}}{8}\left((x-1)^{8}-1\right)-\frac{\alpha^{3} \alpha_{1} m^{9}}{10}\left((x-1)^{10}-1\right)
\end{aligned}
$$

Hence, the series solution in general gives

$$
\begin{aligned}
v(x)=1 & +\frac{m}{2}\left((x-1)^{2}-1\right)+\frac{\alpha m^{3}}{4}\left((x-1)^{4}-1\right)+\frac{\alpha \alpha_{3} m^{5}}{6}\left((x-1)^{6}-1\right) \\
& +\frac{\alpha^{2} \alpha_{4} m^{7}}{8}\left((x-1)^{8}-1\right)-\frac{\alpha^{3} \alpha_{1} m^{9}}{10}\left((x-1)^{10}-1\right)+\ldots \ldots
\end{aligned}
$$

where $\alpha=\alpha_{2}-\alpha_{1}, \alpha_{3}=2 \alpha_{2}-3 \alpha_{1}, \alpha_{4}=\alpha_{2}-3 \alpha_{1}$

By back substitution of values of dimensionless parameters, we get the solution (31) in dimensionless form as

$$
\begin{aligned}
v_{0}(x)= & V_{0}+\frac{\rho g}{2 \mu}\left(x^{2}-2 \delta x\right)+\frac{\left(\alpha_{2}-\alpha_{1}\right)}{4}\left(\frac{\rho g}{\mu}\right)^{3}\left((x-\delta)^{4}-\delta^{4}\right) \\
& +\frac{\left(\alpha_{2}-\alpha_{1}\right)\left(2 \alpha_{2}-3 \alpha_{1}\right)}{6}\left(\frac{\rho g}{\mu}\right)^{5}\left((x-\delta)^{6}-\delta^{6}\right) \\
& +\frac{\left(\alpha_{2}-\alpha_{1}\right)^{2}\left(\alpha_{2}-3 \alpha_{1}\right)}{8}\left(\frac{\rho g}{\mu}\right)^{7}\left((x-\delta)^{8}-\delta^{8}\right) \\
& -\frac{\alpha_{1}\left(\alpha_{2}-\alpha_{1}\right)^{3}}{10}\left(\frac{\rho g}{\mu}\right)^{9}\left((x-\delta)^{10}-\delta^{10}\right)+\ldots \ldots .
\end{aligned}
$$

Here it should be noted that for $\alpha_{1}=\alpha_{2}=0$, we get solution for the Newtonian fluid [1] and for $\alpha_{2}=0$ and setting the value of $\alpha_{1}$ the above solution reduces to that of the third grade obtained in [14].

\subsection{Flow rate and average film velocity}

The flow rate per unit width is given by the formula

$Q=\int_{0}^{\delta} v(x) d x$

Substituting (32) in (33) and then integrating, we obtain the flow rate for an Oldroyd 8-constant fluid as

$$
\begin{aligned}
Q & =V_{0} \delta-\frac{\rho g}{3 \mu} \delta^{3}-\frac{\left(\alpha_{2}-\alpha_{1}\right)}{5}\left(\frac{\rho g}{\mu}\right)^{3} \delta^{5}-\frac{\left(\alpha_{2}-\alpha_{1}\right)\left(2 \alpha_{2}-3 \alpha_{1}\right)}{7}\left(\frac{\rho g}{\mu}\right)^{5} \delta^{7} \\
& -\frac{\left(\alpha_{2}-\alpha_{1}\right)^{2}\left(\alpha_{2}-3 \alpha_{1}\right)}{9}\left(\frac{\rho g}{\mu}\right)^{7} \delta^{9}+\frac{\alpha_{1}\left(\alpha_{2}-\alpha_{1}\right)^{3}}{11}\left(\frac{\rho g}{\mu}\right)^{9} \delta^{11}
\end{aligned}
$$

The average velocity $\bar{V}$ is given by 


$$
\begin{aligned}
\bar{V} & =\frac{Q}{\delta} \\
& =V_{0}-\frac{\rho g}{3 \mu} \delta^{2}-\frac{\left(\alpha_{2}-\alpha_{1}\right)}{5}\left(\frac{\rho g}{\mu}\right)^{3} \delta^{4}-\frac{\left(\alpha_{2}-\alpha_{1}\right)\left(2 \alpha_{2}-3 \alpha_{1}\right)}{7}\left(\frac{\rho g}{\mu}\right)^{5} \delta^{6} \\
& -\frac{\left(\alpha_{2}-\alpha_{1}\right)^{2}\left(\alpha_{2}-3 \alpha_{1}\right)}{9}\left(\frac{\rho g}{\mu}\right)^{7} \delta^{8}+\frac{\alpha_{1}\left(\alpha_{2}-\alpha_{1}\right)^{3}}{11}\left(\frac{\rho g}{\mu}\right)^{9} \delta^{10}
\end{aligned}
$$

It is observed from Eq.(35) that there will be a net upward flow of liquid if $\bar{V}>0$ which implies that

$$
\begin{gathered}
V_{0}>\frac{\rho g}{3 \mu} \delta^{2}+\frac{\left(\alpha_{2}-\alpha_{1}\right)}{5}\left(\frac{\rho g}{\mu}\right)^{3} \delta^{4}+\frac{\left(\alpha_{2}-\alpha_{1}\right)\left(2 \alpha_{2}-3 \alpha_{1}\right)}{7}\left(\frac{\rho g}{\mu}\right)^{5} \delta^{6} \\
+\frac{\left(\alpha_{2}-\alpha_{1}\right)^{2}\left(\alpha_{2}-3 \alpha_{1}\right)}{9}\left(\frac{\rho g}{\mu}\right)^{7} \delta^{8}-\frac{\alpha_{1}\left(\alpha_{2}-\alpha_{1}\right)^{3}}{11}\left(\frac{\rho g}{\mu}\right)^{9} \delta^{10}
\end{gathered}
$$

Inequality (36) provides a reasonable estimation for the belt speed to lift the Oldroyd 8-constant fluid. It shows that a large belt speed is needed to lift a fluid of small viscosity. As a special case, when $\alpha_{1}=\alpha_{2}=0$, the inequality (36) be comes

$V_{0}>\frac{\rho g}{3 \mu} \delta^{2}$,

which is true for Newtonian fluid [1].

\subsection{Force to hold the belt in position}

The force $F$ per unit width to hold the belt in position can also be determined using the expression for shear stress at the belt surface. In fact, we have the formula

$\frac{F}{W}=\int_{0}^{H}\left(S_{x y}\right) d x$

where $H$ is the length of the belt. Using (13) and (38), we obtain

$\frac{F}{W}=-\rho g \delta H$

Eq.(39) can also be used to determine the length of the belt, once the force per unit width is known.

\section{Solution by ADM}

To apply ADM to our nonlinear equation, first we rewrite it in the following operator form [13, 14]:

$L v(x)=-\alpha_{1}\left(N_{1} v(x)\right)+m \alpha_{2}(x-1)\left(N_{2} v(x)\right)+m(x-1)$,

Where $L=\frac{d}{d x}$ is a linear invertible operator and

$$
\begin{aligned}
& N_{1} v(x)=\left(\frac{d v}{d x}\right)^{3} \\
& N_{2} v(x)=\left(\frac{d v}{d x}\right)^{2}
\end{aligned}
$$

Applying the inverse operator $L^{-1}$ on both sides of Eq.(40), we get 
$L^{-1}(L v(x))=-\alpha_{1} L^{-1}\left(N_{1} v(x)\right)+m \alpha_{2} L^{-1}\left((x-1)\left(N_{2} v(x)\right)\right)+L^{-1}(m(x-1))$

so that

$v(x)=v(0)+L^{-1}(m(x-1))-\alpha_{1} L^{-1}\left(N_{1} v(x)\right)+m \alpha_{2} L^{-1}\left((x-1)\left(N_{2} v(x)\right)\right)$

and using the boundary condition (23) we obtain

$v(x)=1+m\left(\frac{x^{2}}{2}-x\right)-\alpha_{1} L^{-1}\left(N_{1} v(x)\right)+m \alpha_{2} L^{-1}\left((x-1)\left(N_{2} v(x)\right)\right)$

We decompose $v(x)$ and the nonlinear terms $N_{1} v(x)=\left(\frac{d v}{d x}\right)^{3}, N_{2} v(x)=\left(\frac{d v}{d x}\right)^{2}$, respectively, as follows

$$
\begin{aligned}
& v(x)=\sum_{n=0}^{\infty} v_{n}(x) \\
& N_{1} v(x)=\sum_{n=0}^{\infty} A_{n} \\
& N_{2} v(x)=\sum_{n=0}^{\infty} B_{n}
\end{aligned}
$$

The first few terms of the adomian polynomials $A_{n}$ and $B_{n}$ are given by

$$
\begin{aligned}
& A_{0}=\left(\frac{d v_{0}}{d x}\right)^{3}, \\
& A_{1}=3\left(\frac{d v_{0}}{d x}\right)^{2} \frac{d v_{1}}{d x}, \\
& A_{2}=3 \frac{d v_{0}}{d x}\left(\frac{d v_{1}}{d x}\right)^{2}+3\left(\frac{d v_{0}}{d x}\right)^{2} \frac{d v_{2}}{d x} \\
& A_{3}=\left(\frac{d v_{1}}{d x}\right)^{3}+6 \frac{d v_{0}}{d x} \frac{d v_{1}}{d x} \frac{d v_{2}}{d x}+3\left(\frac{d v_{0}}{d x}\right)^{2} \frac{d v_{3}}{d x},
\end{aligned}
$$

and

$$
\begin{aligned}
& B_{0}=\left(\frac{d v_{0}}{d x}\right)^{2}, \\
& B_{1}=2\left(\frac{d v_{0}}{d x}\right)\left(\frac{d v_{1}}{d x}\right), \\
& B_{2}=2\left(\frac{d v_{0}}{d x}\right)\left(\frac{d v_{2}}{d x}\right)+\left(\frac{d v_{1}}{d x}\right)^{2}, \\
& B_{3}=2\left(\frac{d v_{0}}{d x}\right)\left(\frac{d v_{3}}{d x}\right)+2\left(\frac{d v_{1}}{d x}\right)\left(\frac{d v_{2}}{d x}\right),
\end{aligned}
$$

We identify the zeroth component $v_{0}(x)$ by

$v_{0}(x)=1+\frac{m}{2}\left((x-1)^{2}-1\right)$,

And the remaining components $v_{n+1}(x)$ by the recurrence relation 
$v_{n+1}(x)=-\alpha_{1} L^{-1} \sum_{n=0}^{\infty} A_{n}+m \alpha_{2} L^{-1}\left((x-1) \sum_{n=0}^{\infty} B_{n}\right), n \geq 0$

Using the same algorithms as are used in [7-8], we obtain the following components

$$
\begin{aligned}
& v_{1}(x)=\frac{\left(\alpha_{2}-\alpha_{1}\right) m^{3}}{4}\left((x-1)^{4}-1\right), \\
& v_{2}(x)=\frac{\left(\alpha_{2}-\alpha_{1}\right)\left(2 \alpha_{2}-3 \alpha_{1}\right) m^{5}}{6}\left((x-1)^{6}-1\right), \\
& v_{3}(x)=\frac{\left(\alpha \alpha_{3}^{2}+\alpha^{2} \alpha_{4}\right) m^{7}}{8}\left((x-1)^{8}-1\right), \\
& v_{4}(x)=\frac{\left(3 \alpha^{2} \alpha_{3} \alpha_{4}+\alpha \alpha_{3}^{2}-\alpha_{1} \alpha^{3}\right) m^{9}}{10}\left((x-1)^{10}-1\right)
\end{aligned}
$$

and so on. In this manner, the rest of the terms in the decomposition series can be calculated.

Summing up, we write the solution in the decomposition series form

$v(x)=v_{0}+v_{1}+v_{2}+v_{3}+\ldots$

This, after inserting the values of $v_{0}, v_{1}, v_{2}$, and $v_{3}$ from (51)-(54), be comes

$$
\begin{aligned}
v(x)= & 1+\frac{m}{2}\left((x-1)^{2}-1\right)+\frac{\alpha m^{3}}{4}\left((x-1)^{4}-1\right)+\frac{\alpha \alpha_{3} m^{5}}{6}\left((x-1)^{6}-1\right) \\
& +\frac{\left(\alpha \alpha_{3}^{2}+\alpha^{2} \alpha_{4}\right) m^{7}}{8}\left((x-1)^{8}-1\right)+\frac{\left(3 \alpha^{2} \alpha_{3} \alpha_{4}+\alpha \alpha_{3}^{2}-\alpha_{1} \alpha^{3}\right) m^{9}}{10}\left((x-1)^{10}-1\right)+\ldots .
\end{aligned}
$$

Here, $\alpha=\alpha_{2}-\alpha_{1}, \alpha_{3}=2 \alpha_{2}-3 \alpha_{1}, \alpha_{4}=\alpha_{2}-3 \alpha_{1}$.The solution of the corresponding problem for the third grade fluid is obtained by setting $\alpha_{2}=0$ and $\alpha_{1}=2 \beta$ [14] and that for the Newtonian fluid can be obtained by setting $\alpha_{1}=\alpha_{2}=0$.

\section{Discussion of results}

Tables 1 and 2 are prepared to show a numerical comparison between three iteration solution of VIM and the four component solution obtained by ADM for various values of the flow parameters $\alpha_{1}, \alpha_{2}$ and $m$. This comparison shows that the numerical results of these methods are in close agreement and they provide realistic series solutions. It is also evident that the errors between the solutions can be reduced further and high accuracy can be achieved by evaluating more components of $v(x)$. Tables 3 and 4 provide a comparison among the approximate solutions $v_{1}(x), v_{2}(x)$ and $v_{3}(x)$ obtained by VIM for different values of $\alpha_{1}, \alpha_{2}$ and $m$. From these tables, we see that for the assigned values of the pertinent flow parameters, VIM gives excellent iterative solutions from one to the next iteration. It is remarkable to observe that third iteration of VIM is almost equivalent to the four terms of ADM solution.

Figs. 1-3 show a comparison between three iteration solution of VIM and the four terms of the ADM solution for different values of $\alpha_{1}, \alpha_{2}$ and $m$ and no visible difference is observed. The quantitative effects of the material parameters $\alpha_{1}$ and $\alpha_{2}$ keeping $m$ fixed on the velocity profile obtained by VIM are observed physically through Figs. 4 and 5. It can be seen from Fig. 4 that for an Oldroyd 8-constant fluid, when the material parameter $\alpha_{1}$ increases from $\alpha_{1}=0.1$ to 1.5 for fixed values of $\alpha_{2}$ and $m$, the fluid velocity increases. However, an almost opposite behavior is observed when $\alpha_{2}$ increases from $\alpha_{2}=0.1$ to 1.5 for $\alpha_{1}=1.0$ and $m=0.5$. Thus, as expected the variation of $\alpha_{1}$ leads to a shear-thinning behavior for fixed $\alpha_{2}$ while the variation in $\alpha_{2}$ leads to a shear-thickening property when $\alpha_{1}$ is fixed. 


\section{Concluding remarks}

Our main goal in this work is to discuss the thin film flow of an Oldroyd 8-constant fluid on a vertical moving belt. The governing nonlinear equation is solved by using VIM. In order to verify the efficiency of this method, the same problem is also solved by ADM. A very good agreement between the results of VIM and those obtained by ADM is observed, which confirm the validity of VIM. In comparison with the results of ADM, one can see that the three terms approximations of the VIM is more effective than three terms solution of ADM. Moreover, the series solution obtained by VIM converges faster than that by ADM and there is less computation work needed in comparison with the Adomian decomposition method. An estimation of the belt speed required to lift the fluid is also recorded. This estimation can be used for experimental verification.

\section{References}

[1] B.R.Munson, D.F. Young, Fundamentals of fluid Mechanics, second edition, John Wiley and Sons, 1994.

[2] A. M. Siddiqui, R. Mehmood, Q.K. Ghori, "Homotopy perturbation method for thin film flow of a third grade fluid down an inclined plane", Chaos, Solitons and Fractals 35 (2008) 140-147.

[3] A. M. Siddiqui, M. Ahmed, Q.K. Ghori, “ Thin film of non-Newtonian fluids on a moving belt”, Chaos, Solitons and Fractals 33 (2007) 10061016.

[4] S. J. Liao, Beyond perturbation: introduction to homotopy analysis method, Chapman \& Hall/CRC Press, Boca Raton, (2003.

[5] J.H. He, "A coupling method of homotopy and perturbation techniques for nonlinear problems", Inter. J. Nonlinear Mech. 35 (2000), 527539.

[6] G. Adomian, Solving Frontier Problems of Physics: The Decomposition Method, Kluwer Academic Publishers, Boston, MA, 1994.

[7] A. M. Waswas, “Adomian Decomposition Method for a reliable treatment of the Emden-Fowler equation”, Appl. Math. Comput. 102 (1999) 77-86.

[8] A. M. Siddiqui, M.A. Hameed, B. M. Siddiqui, "Use of Adomian decomposition method in the study of parallel flow of a third grade fluid", Commun. Nonlinear Sci. Numer Simulat. 15(2012) 2388-2399.

[9] J. H. He, "Variational iteration method-a kind of non-linear analytical technique: some examples", Internal. J. Non-linear Mech. 34(1999) 699-708.

[10] B. Batiha, M.S.M. Noorani, I. Hashim, "Numerical simulation of the generalized Huxley equation by He's variational iteration method", Applied Mathematics and Computation 186 (2007) 1322-1325.

[11] Abdul M. Siddiqui, Ali A. Farooq, Tahira Haroon, “ Application of He's Variational Iteration Method for solvingTthin Film Flow Problem Arising in non-Newtonian Fluid Mechanics", World Journal of Mechanics, 2 (2012), 138-142.

[12] A. M. Siddiqui, A. A. Farooq, T. Haroon, B. S. Babcock, " A comparison of Variational Iteration and Adomian Decomposition Methods in solving Nonlinear Problems”, Applied Mathematical Sciences, 6(2012) 4911-4919.

[13] J.I. Ramos, "On the variational iteration method and other iterative techniques for nonlinear differential equations", Appl. Math. Comput. 199(2008) 39-69.

[14] K Abbaoui, Y. Cherruault, “Convergence of Adomian’s method applied to nonlinear equations”, Math. Comput. Model. 20(1994) 69-73.

[15] Muhammad Yousaf Malik et al, "Analytical Treatment of an Oldroyd 8-constants fluid Between coaxial cylinders with variable viscosity", Commu.Theor. phys., 56(2011) 933-938 


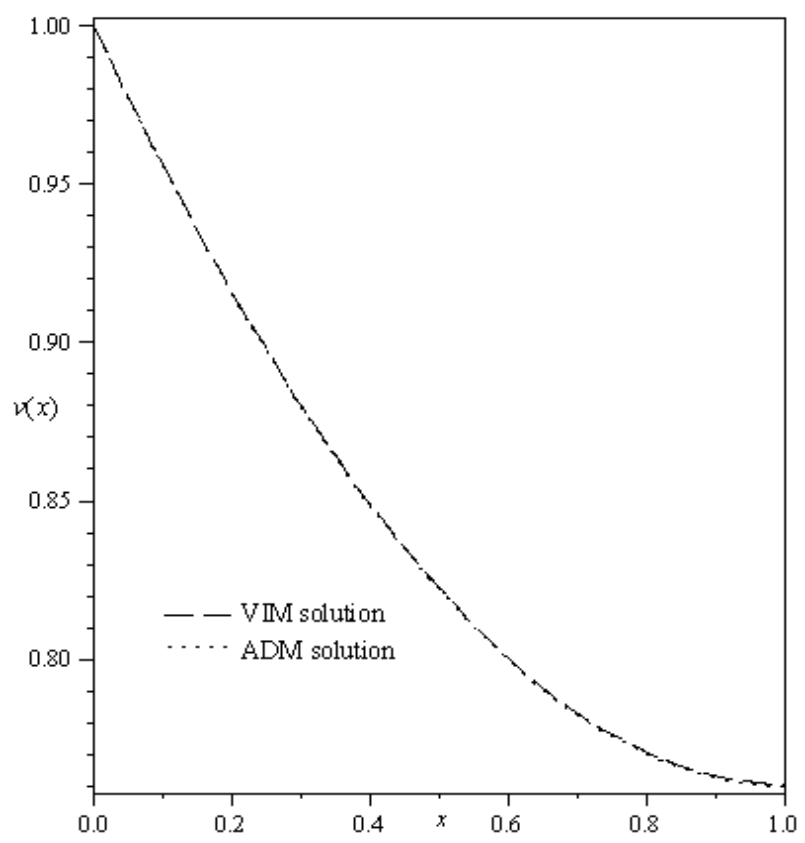

Fig. 1: Comparison of VIM solution and ADM solution for $\alpha_{1}=0.5, \alpha_{2}=0.1, m=0.5$.

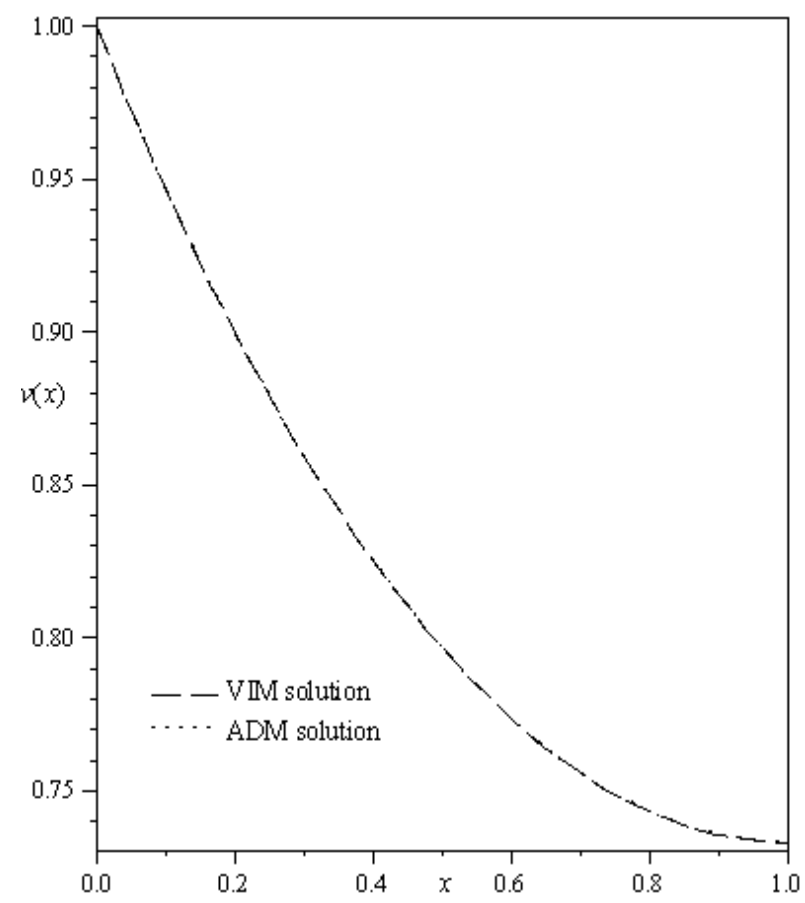

Fig. 2: Comparison of VIM solution and ADM solution for $\alpha_{1}=0.5, \alpha_{2}=1.0, m=0.5$. 


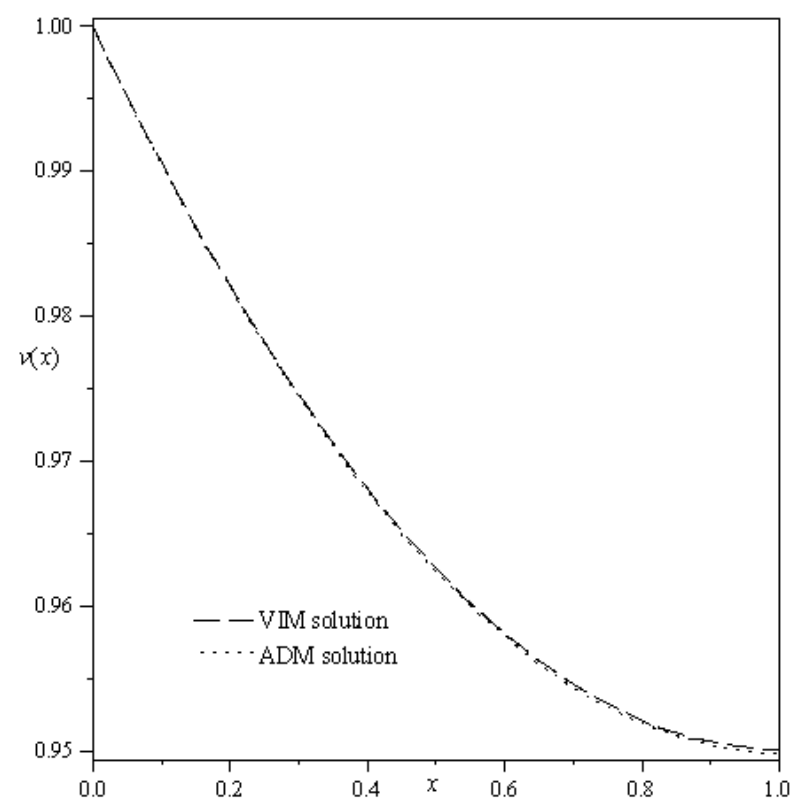

Fig. 3: Comparison of VIM solution and ADM solution for $\alpha_{1}=1.0, \alpha_{2}=0.5, m=0.1$.

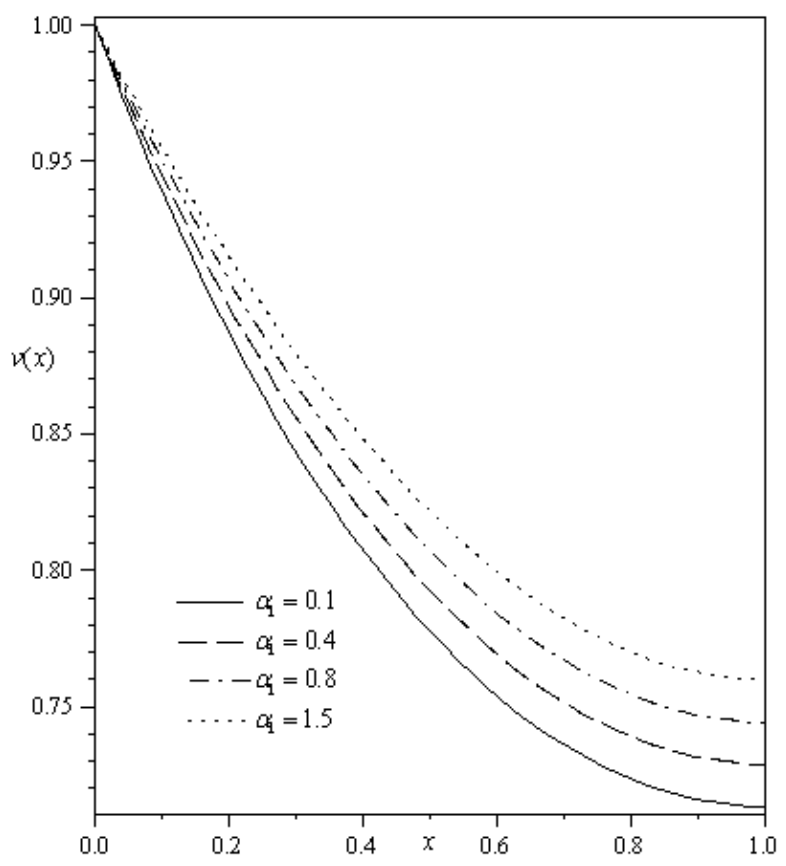

Fig. 4: Effect of $\alpha_{1}$ on velocity profile when $\alpha_{2}=1.0, m=0.5$. 


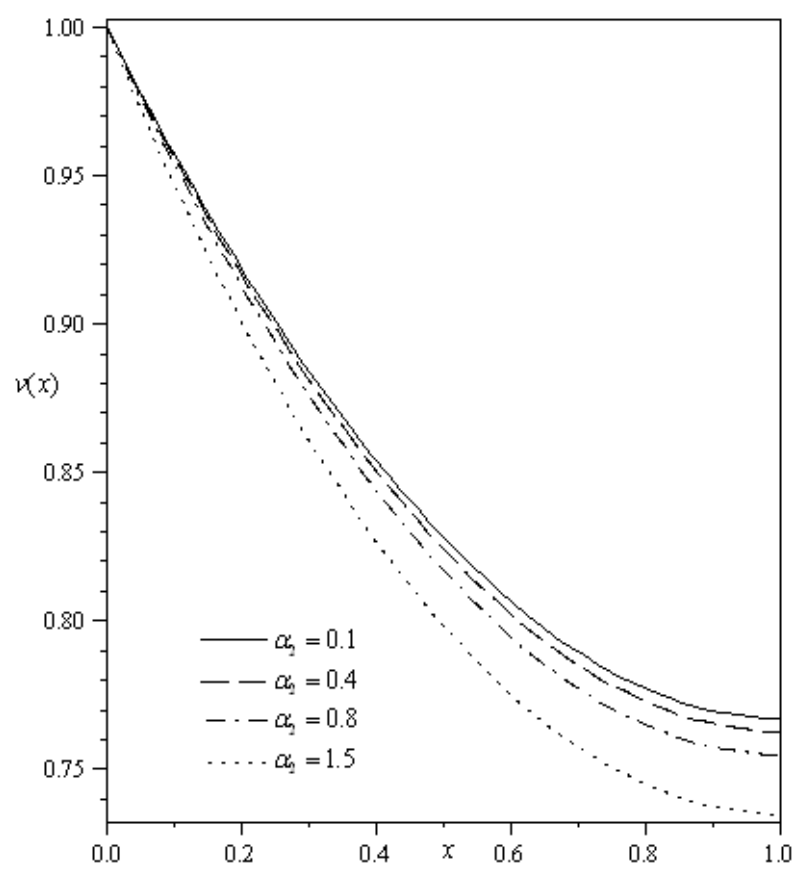

Fig. 5: Effect of $\alpha_{2}$ on velocity profile when $\alpha_{1}=1.0, m=0.5$.

Table1. Comparison between VIM and ADM for $\alpha_{1}=0.1, \alpha_{2}=0.5, m=0.1$

Table 1: Comparison between VIM and ADM for $\alpha_{1}=0.1, \alpha_{2}=0.5, m=0.1$

\begin{tabular}{cccc}
\hline$x$ & VIM solution & ADM solution & Error( VIM-ADM) \\
\hline 0.0 & 1.0000000000 & 1.000000000 & 0.0000000 \\
0.1 & 0.9904653911 & 0.9904653892 & $0.1410 \mathrm{E}-8$ \\
0.2 & 0.9819406154 & 0.981940613 & $0.2110 \mathrm{E}-8$ \\
0.3 & 0.9744235978 & 0.9744235955 & $0.2310 \mathrm{E}-8$ \\
0.4 & 0.9679125147 & 0.9679125123 & $0.2410 \mathrm{E}-8$ \\
0.5 & 0.9624057902 & 0.9624057878 & $0.2410 \mathrm{E}-8$ \\
0.6 & 0.9579020948 & 0.9579020924 & $0.2410 \mathrm{E}-8$ \\
0.7 & 0.9544003433 & 0.9544003409 & $0.2410 \mathrm{E}-8$ \\
0.8 & 0.9518996930 & 0.9518996906 & $0.2410 \mathrm{E}-8$ \\
0.9 & 0.9503995429 & 0.9503995405 & $0.2410 \mathrm{E}-8$ \\
1.0 & 0.9498995329 & 0.9498995304 & $0.2510 \mathrm{E}-8$ \\
\hline
\end{tabular}

Table 2: Comparison between VIM and ADM for $\alpha_{1}=0.5, \alpha_{2}=0.1, m=0.5$

\begin{tabular}{cccc}
\hline$x$ & VIM solution & ADM solution & Error( VIM-ADM) \\
\hline 0.0 & 1.0000000000 & 1.000000000 & 0.0000000000 \\
0.1 & 0.9905339855 & 0.9905339903 & $-0.4810 \mathrm{E}-8$ \\
0.2 & 0.9820584028 & 0.9820584099 & $-0.7110 \mathrm{E}-8$ \\
0.3 & 0.9745752279 & 0.9745752359 & $-0.8010 \mathrm{E}-8$ \\
0.4 & 0.9680862166 & 0.9680862249 & $-0.8310 \mathrm{E}-8$ \\
0.5 & 0.9625928997 & 0.9625929081 & $-0.8410 \mathrm{E}-8$ \\
0.6 & 0.9580965797 & 0.9580965881 & $-0.8410 \mathrm{E}-8$ \\
0.7 & 0.9545983268 & 0.9580965881 & $-0.8410 \mathrm{E}-8$ \\
0.8 & 0.9520989762 & 0.9520989846 & $-0.8410 \mathrm{E}-8$ \\
0.9 & 0.9505991261 & 0.9505991345 & $-0.8410 \mathrm{E}-8$ \\
1.0 & 0.9500991361 & 0.9500991446 & $-0.8510 \mathrm{E}-8$ \\
\hline
\end{tabular}


Table 3: A Comparison among $v_{1}(x), v_{2}(x)$ and $v_{3}(x)$ obtained by VIM for $\alpha_{1}=0.5, \alpha_{2}=0.1, m=0.1$

\begin{tabular}{cccc}
\hline $\boldsymbol{x}$ & $v_{1}(x)$ & $v_{2}(x)$ & $v_{3}(x)$ \\
\hline 0.0 & 1.00000000 & 1.0000000000 & 1.0000000000 \\
0.1 & 0.99050000 & 0.9904570125 & 0.9904568180 \\
0.2 & 0.98200000 & 0.9819262000 & 0.9819258939 \\
0.3 & 0.97450000 & 0.9744050125 & 0.9744046464 \\
0.4 & 0.96800000 & 0.9678912000 & 0.9678908043 \\
0.5 & 0.96250000 & 0.9623828125 & 0.9623824039 \\
0.6 & 0.95800000 & 0.9578782000 & 0.9578778666 \\
0.7 & 0.95450000 & 0.9543760125 & 0.9543755977 \\
0.8 & 0.95200000 & 0.9518752000 & 0.9518747850 \\
1.0 & 0.95050000 & 0.9503750125 & 0.9503754971 \\
\hline
\end{tabular}

Table 4: A Comparison among $v_{1}(x), v_{2}(x)$ and $v_{3}(x)$ obtained by VIM for $\alpha_{1}=1.0, \alpha_{2}=0.5, m=0.1$

\begin{tabular}{cccc}
\hline $\boldsymbol{x}$ & $v_{1}(x)$ & $v_{2}(x)$ & $v_{3}(x)$ \\
\hline 0.0 & 1.00000000 & 1.0000000000 & 0.000000000 \\
0.1 & 0.99050000 & 0.9820738000 & 0.990542211 \\
0.2 & 0.98200000 & 0.9745949075 & 0.982072576 \\
0.3 & 0.97450000 & 0.9681088000 & 0.974593524 \\
0.4 & 0.96800000 & 0.9626171800 & 0.968107218 \\
0.5 & 0.96250000 & 0.9512180000 & 0.962615554 \\
0.6 & 0.95800000 & 0.9546239875 & 0.958120148 \\
0.7 & 0.95450000 & 0.9521248000 & 0.954622329 \\
0.8 & 0.95200000 & 0.9506249875 & 0.952123141 \\
1.0 & 0.95050000 & 0.9501250000
\end{tabular}

